\title{
Nos divertimos y aprendemos con Kahoot! en las clases de Estadística
}

\section{R.M. Alcover, A. Calduch-Losa, S. Vidal-Puig}

Departamento de Estadística e Investigación Operativa Aplicadas y Calidad

Universitat Politècnica de València

\{ralcover, mcalduch, svidalp\}@eio.upv.es

\begin{abstract}
In this work we propose the use of different educational gaming strategies as a tool for learning and working the different generic skills competences. These methodologies have been applied in several statistics subjects delivered in different engineering degrees at the Universitat Politècnica de València (UPV). In order to design the different innovation activities we have chosed the game-based platform Kahoot! based on his straightforward and versatile use. The activities developed by the authors allow to improve the students assistance, the autonomus learning of the students and generic skills development.
\end{abstract}

Keywords: Statistics; Gaming; Educational Innovation; Active Learning; Soft skills; Kahoot!.

\begin{abstract}
Resumen
En el trabajo se aborda la utilización de las estrategias de juego como herramientas de aprendizaje y de trabajo de competencias en varias asignaturas de Estadística impartidas en diferentes Grados de Ingeniería de la Universitat Politècnica de València (UPV). Para el diseño de las actividades de innovación propuestas se ha utilizado la plataforma Kahoot!, por su sencillez y versatilidad de uso. Estas actividades desarrolladas por los autores permiten establecer diferentes dinámicas de trabajo de los alumnos, motivar la asistencia y el trabajo autónomo de los estudiantes, así como desarrollar diferentes competencias.
\end{abstract}

Palabras clave: Estadística; Gamificación; Innovación educativa; Dinamización de aula; Competencias transversales; Kahoot!.

\section{Introducción}

Una de las preocupaciones actuales del profesorado universitario es conseguir y mantener la motivación e interés de los estudiantes en sus clases. Esta preocupación se incrementa en aquellas asignaturas que cumplen estas dos condiciones: están ubicadas en los primeros cursos de las titulaciones y no están directamente relacionadas con la titulación en la que se imparten. Son las asignaturas que constituyen las materias básicas de los actuales Grados. Este es el caso de las asignaturas de la materia Estadística, impartida en primer o segundo curso de los diferentes Grados de Ingeniería en la Universitat Politècnica de València (UPV). 
En algunas clases de estas asignaturas es difícil mantener la atención del estudiante durante toda la sesión (90 minutos), sobre todo cuando en los horarios establecidos por las correspondientes Escuelas (ERT) estas sesiones tienen lugar a últimas horas de la tarde. Los alumnos, tras realizar diferentes actividades durante la mañana (algunos de ellos desempeñan trabajos en empresas y llegan a clase con una jornada laboral ya cumplida), están algo cansados. Como profesorado, no debemos plantearnos estas cirscunstancias como un problema, sino como un reto que nos motive y estimule a organizar nuestras clases de la mejor manera posible para adaptarnos a las diferentes situaciones que se nos puedan plantear. Debemos actualizarnos constantemente tanto en conocimientos de nuestra área, como en la metodología empleada para conseguir desarrollar en nuestros alumnos las competencias que les conducirán, a su vez, a ser buenos profesionales (GIMA, 2008).

La introducción en el aula de elementos o actividades de juego es un tipo de gamificación (Kapp, 2012), esto es, una técnica de aprendizaje que traslada la mecánica de los juegos al ámbito educativo con el fin de conseguir mejores resultados, constituyendo una experiencia positiva para el alumno y el profesor (EDUCACIÓN 3.0). Numerosos trabajos confirman los buenos resultados obtenidos tras introducir estrategias de juego en el ámbito docente, como ejemplo Del Cerro (2015), Villalustre y Moral (2015), Rodriguez-Fernández (2017).

Por otra parte, en los últimos años con el desarrollo de las TIC (Tecnologías de la información y comunicación), se han introducido en la docencia sistemas que intentan estimular la participación activa de los alumnos mediante sistemas de respuesta personal (Personal Response System o PRS). Estos sistemas, inicialmente denominados “clickers", son unos dispositivos con unos mandos electrónicos que pueden utilizarse para la realización de preguntas e interpretación de resultados en tiempo real. El avance de la tecnología y la proliferación de los dispositivos móviles ha dejado obsoletos los anteriores sistemas, siendo los teléfonos inteligentes o smartphones (también puede utilizarse cualquier tableta o PC) los elementos clave en la introducción de las TIC en el proceso educativo. Actualmente todos los alumnos disponen de uno de estos teléfonos, que se han convertido en potentes herramientas educativas y que nos pueden proporcionar múltiples ventajas (Fuertes, 2016). La disponibilidad en las aulas de internet y plataformas como Kahoot!, Socrative, QuestionPress, Google forms, Brainscape, Cerebriti, Knowre, etc... combinan la facilidad y rapidez de uso de estos dispositivos, bajo mantenimiento y coste nulo.

Para la innovación que presentamos en este trabajo, el grupo de profesores implicado ha seleccionado la plataforma on line Kahoot! (https://kahoot.com), desarrollada en 2013 por el profesor Alf Inge Wang de la Norwegian University Of Science And Technology. Son numerosos los trabajos que hacen referencia a esta herramienta, como ejemplo Dellos (2015), Johns (2015) y Plump y LaRosa (2017). En nuestro caso, las actividades que introducimos en el aula desde las diferentes asignaturas de Estadística en la UPV programadas con Kahoot! nos van a permitir, además de las ventajas ya referidas de este tipo de sistemas, el poder trabajar y hacer hincapié en las diferentes competencias que nuestras asignaturas tienen establecidas. No perdamos de vista que la introducción de las 
TIC facilita al profesorado múltiples actividades didácticas, permitiendo adaptarse más fácilmente al tipo de alumnado y mejorar su aprendizaje.

En nuestra opinión, este tipo de estrategias de juego combinadas con las nuevas tecnologías, posee un gran potencial como herramientas para mejorar el aprendizaje e incrementar el trabajo competencial en el aula, como mostramos con la descripción de las actividades que presentamos en este trabajo. De forma análoga queremos incidir en la facilidad y versatilidad del uso de Kahoot!, lo que incrementa su capacidad de establecer diferentes dinámicas de trabajo activas en el aula, generando múltiples posibilidades y redundando todo ello en una valiosa herramienta de aprendizaje significativo, tal como mostramos en los siguientes apartados.

\section{Objetivos}

El propósito general de nuestro trabajo ha sido explorar el uso de la plataforma Kahoot! para realizar diferentes tipos de actividades en el aula que involucren activamente al alumnado y fomenten el aprendizaje. Algunas de las actividades que hemos diseñado se han llevado a cabo por primera vez en la docencia de las asignaturas del área de Estadística en nuestra universidad. Más en detalle, los objetivos que se pretenden con nuestra propuesta son los siguientes:

- Utilizar un juego como herramienta de trabajo en el aula, implicando y motivando al alumno en el proceso de aprendizaje.

- Captar la atención del alumno, incrementar su participación y conseguir un aprendizaje activo.

- Trabajar con el juego algunas competencias transversales.

- Implicar en el seguimiento de la clase a los alumnos más tímidos y/o con ciertas minusvalías.

- Reforzar el aprendizaje resumiendo y resaltando conceptos relevantes de cada unidad didáctica.

- Evaluar algunos contenidos de conocimiento de las unidades didácticas.

Los profesores implicados pretendemos conseguir estos objetivos involucrando al alumno en el "juego" (actividad), de tal manera que él piense que está jugando en lugar de estudiando. Es decir, que no sea consciente de que está trabajando, simplemente con este tipo de actividades el estudiante se divierte, como un niño. La mecánica del juego, las imágenes que presentamos junto con las preguntas, el formato de elección de las respuestas...., transforma una tarea o evaluación, digamos formal, en una actividad lúdica en la que los alumnos interactúan, comentan, se mueven, ríen y en defintiva, se divierten aprendiendo. Como docentes, es reconfortante ver a nuestros estudiantes trabajando activamente los contenidos. 


\section{Desarrollo de la innovación}

Las actividades con la plataforma Kahoot! se han llevado a cabo en asignaturas de Estadística de diferentes Grados impartidos en la Universitat Politècnica durante el curso académico 2017-2018: Grado de Ingeniería Informática (GII), Grado en Ciencia y Tecnología de los Alimentos (GCTA), Grado en Ingeniería en Diseño Industrial y Desarrollo de Productos (GIDDP), Grado en Ingeniería Forestal y del Medio Natural (GIFMN).

\subsection{Innovación 1}

En la asignatura de Estadística (primer curso del GII) la plataforma se ha utilizado diseñando actividades con diferentes objetivos. Uno de ellos ha consistido en utilizar Kahoot! como refuerzo de aprendizaje al final de la clase. En el GII todas las sesiones impartidas tienen una duración de 90 minutos. Tras 60 minutos de trabajo y dedicación del alumno a las explicaciones del profesor, disminuye su nivel de atención. El cambio de actividad y la novedad del juego permite de nuevo recuperar la atención del alumno. Este tipo de actividades que hemos llevado a cabo ha tenido una duración aproximada de 30 minutos en cada sesión.

Por ejemplo, en la unidad didáctica de Introducción al Cálculo de Probabilidades del temario de la asignatura, se ha planteado un cuestionario (Figura 1) con el fin de revisar conceptos, reforzar conocimientos y trabajar diferentes competencias. Especialmente relevantes resultan las competencias transversales (http: //www.upv.es/contenidos/COMPTRAN) .

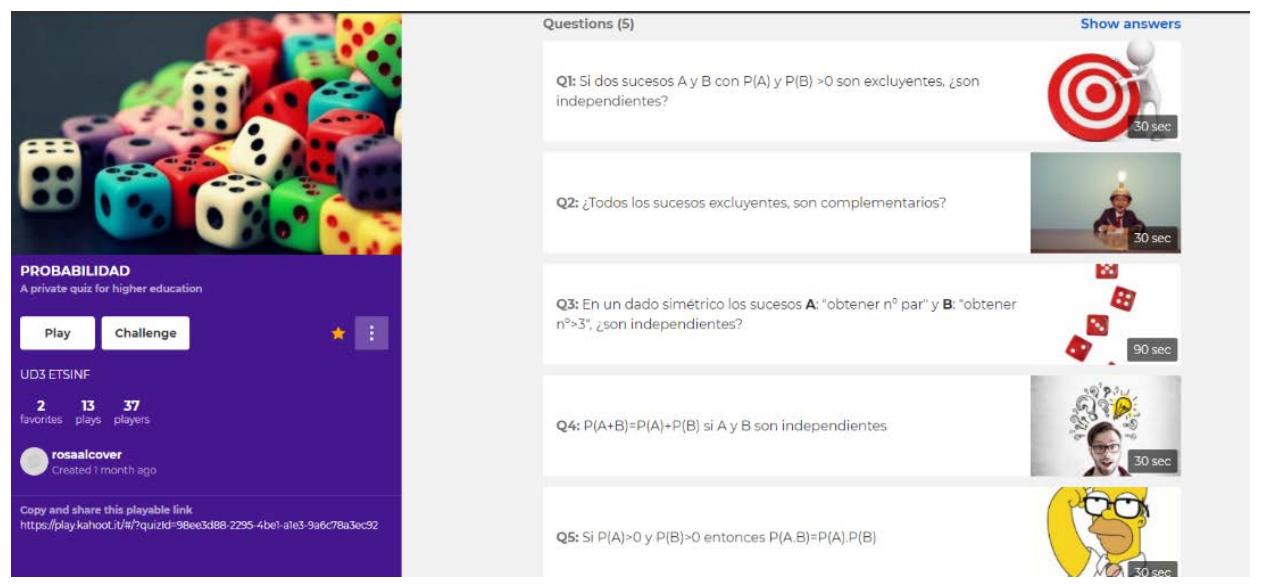

Fig. 1 Cuestionario con cinco preguntas de la Unidad de Cálculo de Probabilidades

Los alumnos, de manera individual se conectan a través de sus teléfonos móviles a la plataforma. Tras ir respondiendo las preguntas y obtener las puntuaciones finales de cada uno de ellos, el alumno ganador es ovacionado y recibe el reconocimiento por parte del resto de sus compañeros. A continuación se revisa cada una de las preguntas del cuestionario y entre aquellos que han acertado la respuesta a cada pregunta un alumno, voluntariamente, justifica al resto por qué cada una de las respuestas alternativas es correcta o incorrecta. Se trata de una exposición de los argumentos del alumno, con su lenguaje y

(cc) EY-NC-ND 2018, Universitat Politècnica de València

Congreso IN-RED (2018) 
vocabulario, al resto de los compañeros, generándose un debate productivo. En este caso el profesor actúa de moderador, regulando el turno de las intervenciones entre ellos y gestionando el tiempo.

Además de utilizar esta actividad como refuerzo de aprendizaje (cada alumno reflexiona sobre cada pregunta del cuestionario dos veces) y trabajar en ella diferentes competencias según la naturaleza de la pregunta, se incide especialmente en desarrollar las habilidades de comunicación oral por parte de los alumnos. Este tipo de actividad se ha trabajado de forma sistemática a lo largo de un semestre con un grupo de 26 alumnos matriculados en el turno de tarde.

La competencia transversal de comunicación efectiva (CT-08, Proyecto institucional de las competencias transversales UPV) se trabaja con frecuencia en el aula, en forma escrita: ejercicios, memorias, informes de prácticas, exámenes. Sin embargo, en su dimensión oral se incide con menor frecuencia, por lo menos en primer curso. Con la actividad propuesta intentamos que el alumno transmita "conocimientos" de la manera lo más clara posible, esforzándose en construir correctamente las frases aunque utilice su propio lenguaje. Además, el alumno debe saber responder a las preguntas que durante las explicaciones le formulan sus compañeros. La mayoría de ellos utiliza el lenguaje gestual para apoyar sus explicaciones. Finalmente, con el debate los alumnos elaboran sus propios argumentos, los exponen y los defienden, trabajando esta competencia de manera más compleja.

\subsection{Innovación 2}

La siguiente actuación se realizó también con alumnos de primer curso de GII, pero en otros grupos diferentes al que realizó la actividad de innovación 1 que se acaba de exponer. Estos grupos constan de 20 y 37 alumnos matriculados en el turno de mañana. En este caso se cambió la manera de utilizar Kahoot!, proponiendo a los alumnos que fueran ellos quienes plantearan posibles preguntas para realizar un Kahoot! con posterioridad. Así, los alumnos invertían más tiempo en la experiencia en su casa que en el aula. Lo que se pretende con esta actuación es un aprendizaje activo, al que llamamos ACL (ACtive Learning), ya que se ha conseguido que los alumnos participantes en la experiencia profundizaran en los conocimientos teóricos de la materia, al revisar los contenidos de las unidades temáticas para plantear las cuestiones. Se ha constatado que las preguntas propuestas por los alumnos eran más complicadas que las que normalmente plantea la profesora, ya que parece que los estudiantes "quieren cazar" a sus compañeros, poniendo adrede cuestiones más complicadas. Esto ha provocado un aumento del nivel de los conocimientos en el aula.

La actividad se propone como voluntaria, y los estudiantes que así lo desean envían sus preguntas por correo electrónico a la profesora. En la Figura 2 se muestra una imagen compartida por un estudiante: 


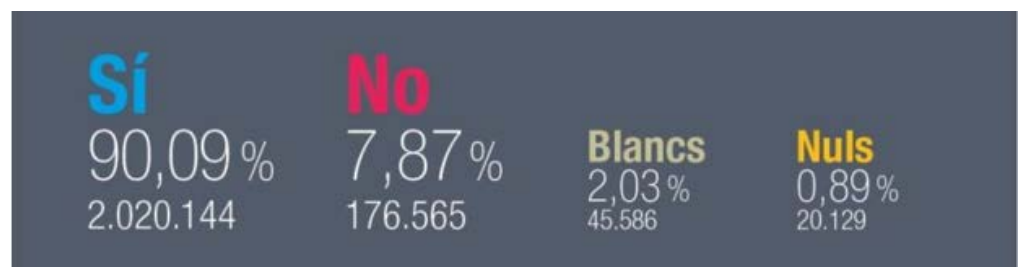

Fig. 2 Imagen presentada por un alumno

La pregunta que hacía el alumno en cuestión era: “¿Cuál es el problema de este porcentaje de resultados?”, y las posibles respuestas que daba el estudiante eran: a) Los porcentajes no suman $100 \%$, b) Solo está bien el porcentaje de nulos, c) Las dos anteriores.

En esta ocasión, la pregunta y las respuestas proporcionadas nos indican que el alumno que las ha enviado tiene claros los conceptos que pregunta y ha buscado o calculado los números adecuados.

Al presentar la pregunta en clase, sirvió para repasar dos temas de la asignatura: Estadística descriptiva y Probabilidad condicional. Por lo tanto, vemos cómo con esta modalidad de Kahoot!, los alumnos comparten conocimiento y tienen un doble refuerzo: cuando piensan e investigan para proponer las preguntas y cuando las responden. Además, hay que destacar que en esta acción se está trabajanado la CT-01 de la UPV, Comprensión e integración, ya que un alumno tiene que comprender las unidades didácticas para proponer sus preguntas (Proyecto institucional de las competencias transversales de la UPV).

\subsection{Innovación 3}

En los grados de GIDDP (asignatura: Matemáticas II), GCTA (asignatura: Bioestadística) y GIFMN (asignatura: Métodos Estadísticos) también se utilizó como una herramienta de refuerzo del aprendizaje de las diferentes unidades temáticas, pero de forma diferente al tipo de innovación 1, anteriormente expuesto. Para ello se realizaban unas 10-15 preguntas al final de cada unidad temática y se daba entre 20 y 30 segundos para responder a cada una de las preguntas, dependiendo de la dificultad de las mismas. Al final de cada pregunta y con la información del número de respuestas obtenido en cada una de las opciones propuestas, y antes de pasar a la pregunta siguiente, se procedía a revisar los fallos más importantes y numerosos, de modo que el alumno pudiera reforzar los conceptos y relaciones más importantes vistas en la unidad temática. Este enfoque permite al alumno darse cuenta de sus errores y se situaría, por tanto, dentro del campo de las evaluaciones con carácter formativo. La actividad venía a tener una duración total de entre 15 y 20 minutos y a parte de potenciar el aprendizaje servía para proporcionar un final de la clase más competitivo y motivador.

A diferencia de la innovación 1, en este caso los cuestionarios presentados a los alumnos son más largos, no se trabaja la competencia de comunicación efectiva y los resultados son tenidos en cuenta en la calificación final del alumno. 


\section{Resultados}

Los resultados presentados corresponden a las asignaturas de Bioestadística (recogidas: 34 encuestas de 69 alumnos), Matemáticas II (recogidas: 20 de 55 alumnos) y Métodos Estadísticos (Met. Estadísticos) (recogidas: 16 encuestas de 32 alumnos). A partir de los resultados de las encuestas pasadas a los alumnos en estas asignaturas, obtenidos al final del primer cuatrimestre, podemos afirmar que el uso del Kahoot! ha tenido muy buena acogida, con una opinión de más del 80\% que consideraba el uso de la herramienta como adecuado o muy adecuado (Figura 3).

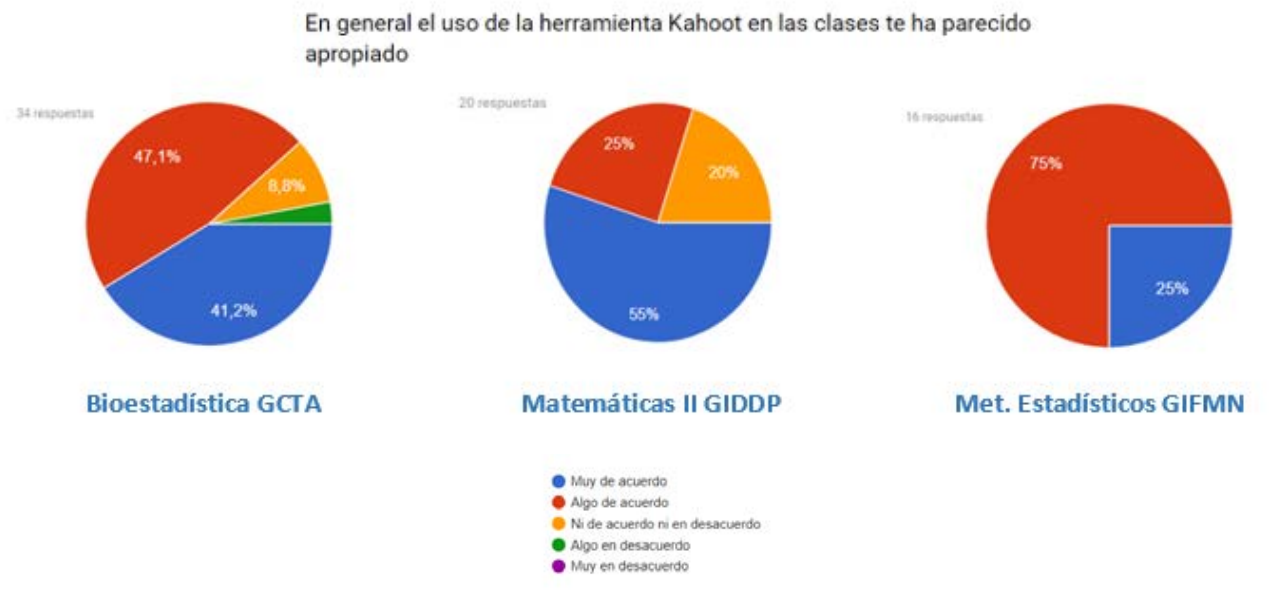

Fig. 3 Resultados de Ia cuestion 1 de la encuesta

En cuanto a la motivación (Figura 4), de nuevo más del 80\% de los alumnos consideraba que mejoraba su motivación.

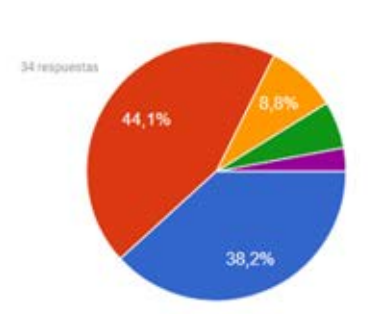

Bioestadística GCTA

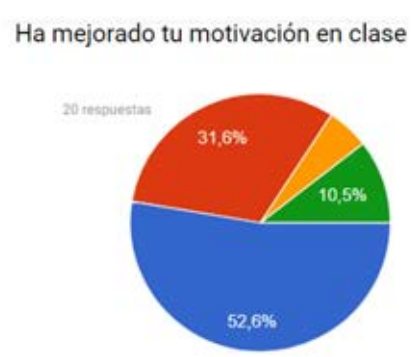

Matemáticas II GIDDP

- Agro de acuerdo

Ans acuerdo ni en desacuerdo

- Algo en dessaverdo

Fig. 4 Resultados de la Cuestión 2 de la encuesta

En relación a la pregunta sobre si se habían divertido con la actividad, más del 85\% de los alumnos estaban de acuerdo en los tres grupos (Figura 5). 


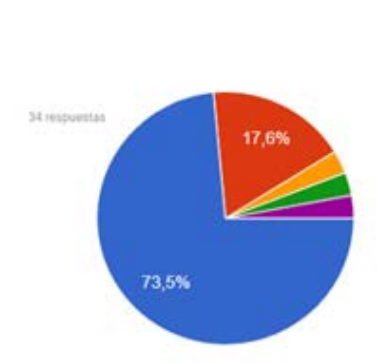

Bioestadística GCTA
Te has divertido con el Kahoot

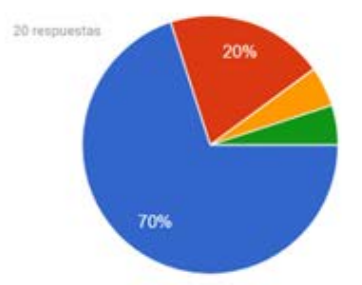

Matemáticas II GIDDP

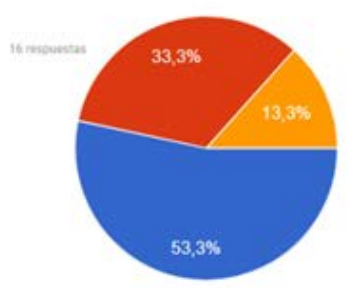

Met. Estadísticos GIFMN

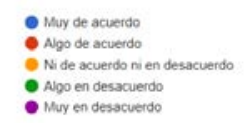

Fig. 5 Resultados de la Cuestión 3 de la encuesta

Respecto a la dificultad de las preguntas realizadas, el 35\% de los alumnos de Matemáticas II, el 52\% de Bioestadística y el 7\% de los de Met. Estadísticos estaban de acuerdo o muy de acuerdo con que eran difíciles (Figura 6).

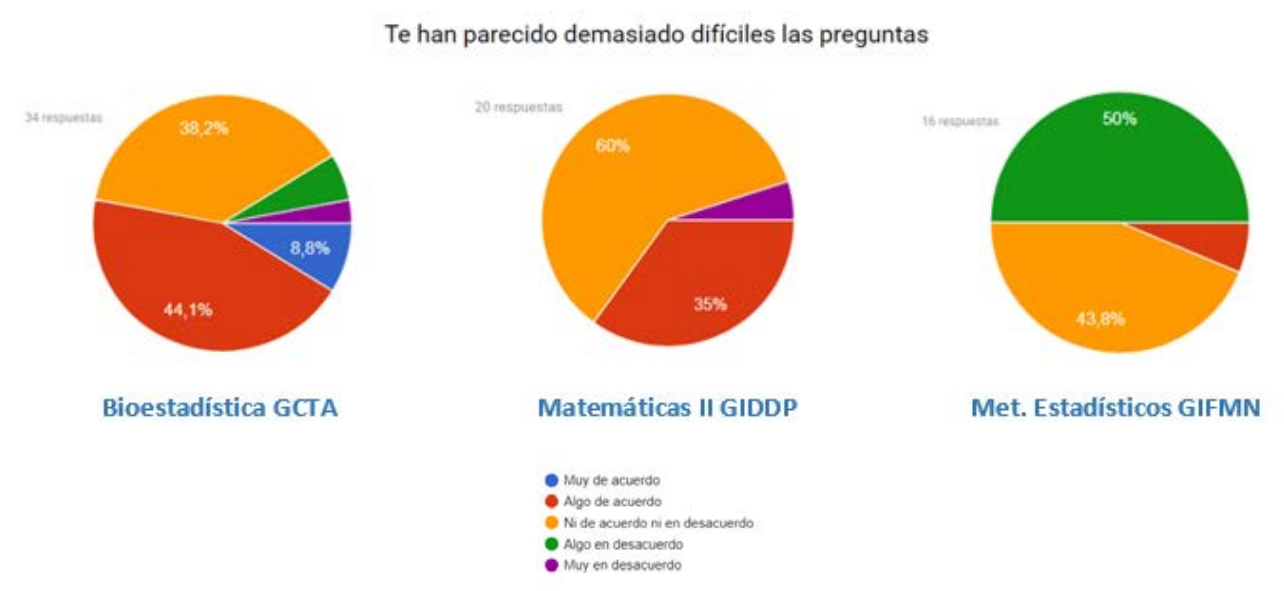

Fig. 6 Resultados de la Cuestión 4 de la encuesta

Respecto a la posible falta de tiempo, un 50\% de los alumnos de Matemáticas II, un 73.5\% de los de Bioestadística y un 62\% de los de Met. Estadísticos estaban de acuerdo (Figura 7).

(cc) BY-NC-ND 2018, Universitat Politècnica de València 
Te ha agobiado la falta de tiempo al contestar

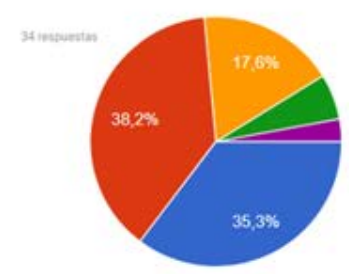

Bioestadística GCTA

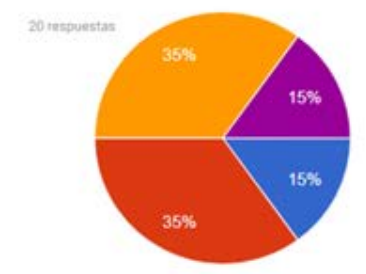

Matemáticas II GIDDP

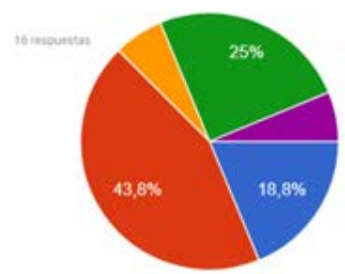

Met. Estadísticos GIFMN

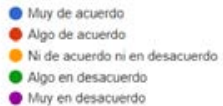

Fig. 7 Resultados de la Cuestión 5 de la encuesta

Hay que indicar que los Kahoot! realizados en Bioestadística eran de mayor dificultad y centrados en herramientas de Inferencia Estadística frente a los de Matemáticas II que estaban centrados en Estadística Descriptiva y Probabilidad. Los alumnos de Met. Estadísticos son los que realizaron más pruebas con Kahoot!, cubriendo Estadística Descriptiva e Inferencia.

Respecto a la opinión de los alumnos en relación el refuerzo del aprendizaje (objetivo principal de la metodología empleada), cabe señalar (Figura 8) que en el caso de Matemáticas II un 65\% de los alumnos estuvo de acuerdo o muy de acuerdo con que la metodología reforzaba su aprendizaje y un $10 \%$ estaba en ligero desacuerdo. En el caso de Bioestadística, un $47.1 \%$ de los alumnos estuvo de acuerdo o muy de acuerdo frente a un 29.4\% que estaba en desacuerdo y, por último, en Met. Estadísticos un 56\% de los alumnos estuvo de acuerdo o muy de acuerdo frente a un $12.5 \%$ que estaba en ligero desacuerdo.

Te ha servido de refuerzo de aprendizaje al acabar los temas

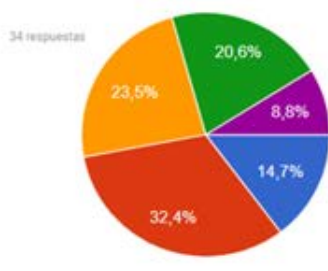

Bioestadística GCTA

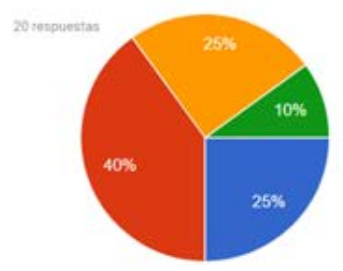

Matemáticas II GIDDP

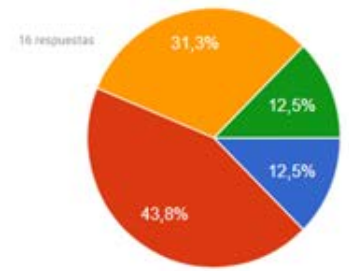

Met. Estadísticos GIFMN

- Mago de acuerdo

Ni de acuerdo ni en desacuerdo

- Algo en desacuerdo

Fig. 8 Resultados de la Cuestión 6 de la encuesta

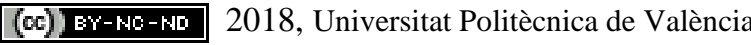

Congreso In-Red (2018) 
Finalmente, en relación a la pregunta sobre si estaban de acuerdo con que se utilizara sus resultados en el Kahoot! para valorar su participación en clase (Figura 9), había más variación en las opiniones, estando en el caso de Matemáticas II el 60\% de los alumnos a favor frente a un $20 \%$ en contra, en Bioestadística del $41.1 \%$ a favor frente al $41.2 \%$ en contra y en Met. Estadísticos el 49.9\% de los alumnos a favor frente a un 31\% en contra.

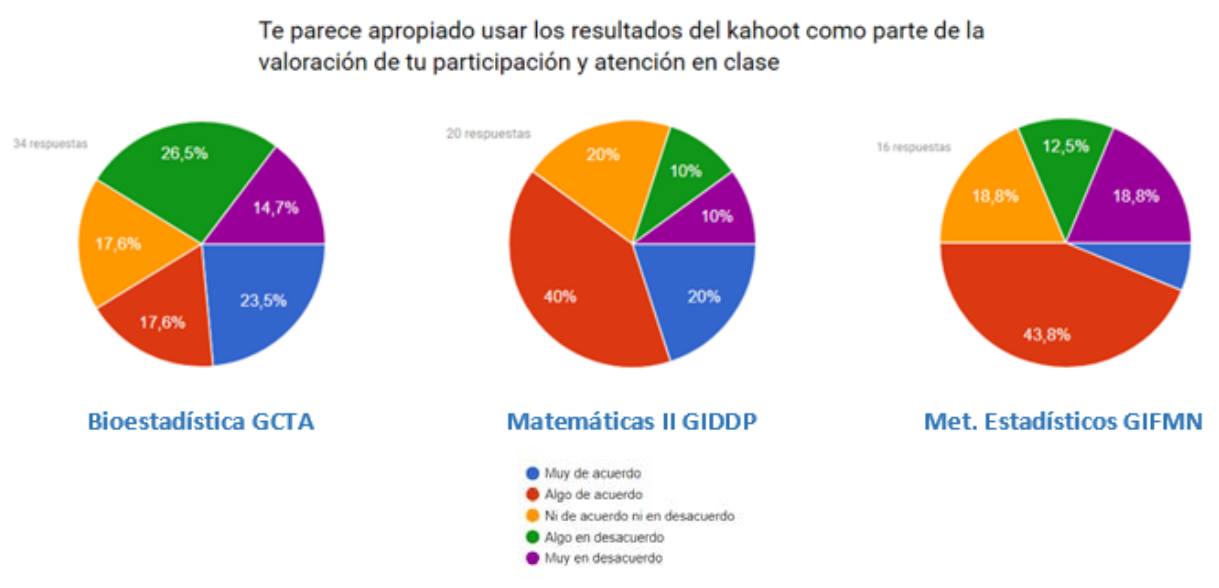

Fig. 9 Resultados de la Cuestión 7 de la encuesta

\section{Conclusiones}

En los últimos años, con el desarrollo de las TIC se han introducido en la docencia sistemas que intentan fomentar la participación activa del alumno en el proceso de enseñanzaaprendizaje. Por otra parte, los resultados de estrategias de juego en las aulas han proporcionado resultados positivos, como han mostrado diversas experiencias previas.

En este trabajo combinamos ambas modalidades utilizando la plataforma Kahoot! con el fin de introducir dinámicas de juego en el aula que estimulen y fomenten la participación activa y el trabajo autónomo de los estudiantes. Para ello, se han propuesto tres actividades de innovación educativa, que además de conseguir estos objetivos generales, han proporcionado las siguientes evidencias: alto grado de motivación y diversión ; el tiempo de respuesta, la dificultad de las preguntas y la posible evaluación de la actividad se perfila globalmente como una cuestión muy relevante para los estudiantes. Señalar que esta cuestión no afecta negativamente a la valoración que hacen los alumnos sobre la idoneidad de las metodologías propuestas.

En la actividad de innovación 3, los resultados ponen de manifiesto que la actividad ha servido realmente para reforzar el aprendizaje de los contenidos de las asignaturas.

Respecto a las actividades de innovación 1 y 2, están actualmente en desarrollo y no se dispone de la valoración final de los alumnos, pero de momento y tras aplicarlo desde el inicio del cuatrimestre, la opinión de los estudiantes y su participación en estas actividades es muy buena. Los resultados provisionales confirman todas las conclusiones expuestas 
hasta el momento. Consideramos interesante encuestar a los alumnos involucrados en estas dos innovaciones y, una vez acabado el curso, comparar los resultados con los obtenidos en la innovación 3. Esta es nuestra próxima tarea.

\section{Referencias}

DEL CERRO GÓMEZ, G. (2015). “Aprender jugando, resolviendo: diseñando experiencias positivas de aprendizaje“. En XII Jornadas Internacionales de Innovación Universitaria (20-21 Julio 2015. Villaviciosa de Odón). Madrid: Universidad Europea de Madrid. 237-244.

DELLOS, R. (2015). "Kahoot! A digital game resource for learning" en International Journal of Instructional Technology and Distance Learning, vol. 12, issue 4, p. 49-52.

EDUCACIÓN 3.0. ¿Qué es la gamificación y cuáles son sus objetivos? https://www.educaciontrespuntocero.com/noticias/gamificacion-que-es-objetivos/70991.html [Consulta: 17 de marzo de 2018].

FUERTES, A. et al. (2016). "Uso de herramientas de respuesta de audiencia en la docencia presencial universitaria. Un primer contacto“. En XXII Jenui (6-8 Julio 2016. Almería) Editorial Universidad de Almería. 261-268.

GIMA (2008). Metodologías activas. Valencia: Universitat Politècnica de València, Editorial UPV.

JOHNS, K. (2015). "Engaging and Assessing Students with Technology: A Review of Kahoot!“ en Delta Gamma Bulletin, vol. 81, issue 4, p. 89

KAPP, K.M. (2012). The gamification of Learning and Instruction : game based Methods and Starategies for Training and Education. Ed. Pfeiffer-Wiley. New York.

PLUMP, C. y LAROSA, J. (2017). "Using Kahoot! in the Classroom to Create Engagement and Active learning: A Game-Based Technology Solution for eLearning Novices“ en Management Teaching Review, vol. 2, issue 2, p. 151-158.

RODRIGUEZ-FERNÁNDEZ, L. (2017). "Smartphone y aprendizaje : el uso de Kahoot en el aula universitaria“ en Revista Mediterránea de Comunicación. Universidad de Alicante (2017, vol.8, núm. 1, pp. 181-190).

UPV, Universidad Politécnica de Valencia. Proyecto institucional de las competencias transversales. http://www.upv.es/contenidos/COMPTRAN/) [Consulta: 14 de marzo de 2018].

VILlALUSTRE, M. L. y MORAL, P. E. (2015). "Gamificación: Estrategia para optimizar el proceso de aprendizaje y la adquisición de competencias en contextos universitarios“. En Digital Education. Revistes Cientifiques de la Universitat de Barcelona (2015, núm. 27, pp. 13-31). http://greav.ub.edu/der [Consulta: 18 de Marzo de 2018] 